\title{
Soledad, impulsividad, consumo de alcohol y su relación con suicidalidad en adolescentes
}

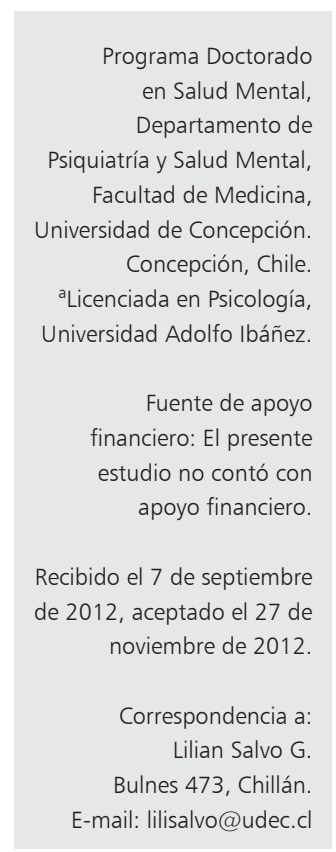

\author{
LILIAN SALVO G., ANDREA CASTRO S. ${ }^{a}$
}

\section{Association of loneliness, impulsivity and alcohol use with suicidal behavior in adolescents}

\begin{abstract}
Background: Suicide and suicide attempts are public health problems. Their prevention requires the detection of predictor factors. Aim: To determine the predictive value of loneliness, impulsivity and alcohol use on suicidal behavior in adolescents. Subjects and Methods: Suicidal behavior, Loneliness (UCLA), Impulsivity (Barratt) scales and the Alcohol Use Disorders Identification Test (AUDIT), were applied to 763 high school students aged 14 to 19 years (49\% males), living in Chillán, Chile. Results: Nineteen percent of participants had attempted suicide and 34.3\% had suicidal ideation. Loneliness, impulsivity and alcohol use were directly related to suicidal behavior. These predictors explained 31\% of the suicidal behavior. The most important risk factor was loneliness, followed by female gender, impulsivity and alcohol use. Conclusions: Loneliness, impulsivity and alcohol use are risk factors for suicide among adolescents. Women are at higher risk than men.
\end{abstract}

(Rev Med Chile 2013; 141: 428-434).

Key words: Adolescent; Suicide attempted; Suicidal ideation.
4 1 suicidio y los intentos de suicidio constituyen un importante problema de salud pública y la Organización Mundial de la Salud pronostica un millón y medio de suicidios en el mundo para el año $2020^{1}$. Durante los últimos 50 años, las tasas mundiales de suicidio han aumentado considerablemente observándose importantes cambios en la distribución por edad, con incremento significativo en adolescentes y adultos jóvenes ${ }^{1-3}$. Pero las cifras acumuladas pueden ocultar diferencias entre algunos segmentos de la población. Además, las tasas varían en cada país y en cada década como respuesta a los múltiples factores que se asocian con la conducta suicida. Por tanto, la explicación precisa en dichas variaciones así como de los factores de riesgo debe ser considerada en el contexto local ${ }^{1-4}$.

A nivel mundial, el suicidio representa la segunda causa de muerte en personas de 10 a 24 años de edad, después de los accidentes de tráfico ${ }^{5}$. Por otro lado, investigaciones en muestras de adolescentes, representativas de realidades nacionales, revelan prevalencias de vida de intentos suicidas que varían entre $1,5 \%$ y $12,1 \%$ y prevalencias de 12 meses de $1,8 \%$ a $8,4 \% \%^{6-8}$. Además, en ese grupo etario se han encontrado prevalencias de vida de ideas suicidas que fluctúan entre $21,7 \%$ y $37,9 \%$, y de planes suicidas de 3,0\%; y prevalencia de 12 meses de $26,0 \%$ para ideas suicidas y $15,0 \%$ para planes suicidas ${ }^{6,8,9}$.

Chile no dispone de tasas de prevalencia de intentos ni de ideas suicidas en la población general a nivel nacional. Sólo cuenta con información proveniente de estudios en adolescentes estudiantes de enseñanza media de algunas comunas y ciudades del país que describen prevalencias de vida de intentos suicidas de entre $14,2 \%$ y $19,0 \%$ y prevalencias en los últimos 12 meses de ideas suicidas (de diferente severidad) de 7 a $62 \%^{10-14}$.

En la actualidad no existen dudas de que la suicidalidad, entendida como un constructo que abarca las ideas suicidas, los intentos de suicidio y el suicidio, está determinada por la interrelación 
de diferentes factores entre los que se incluyen los aspectos demográficos, genéticos, biológicos, psiquiátricos, psicológicos, familiares y sociales ${ }^{15-20}$. Existe un amplio cuerpo de evidencia sobre los factores de riesgo, pero su relación con intentos suicidas e ideas suicidas se ha estudiado generalmente en poblaciones clínicas.

En los estudios efectuados en población general adolescente, a nivel internacional, algunas variables han sido preferentemente estudiadas. Entre ellas destacan género, edad, historia familiar y personal de conducta suicida, trastornos psiquiátricos, funcionamiento familiar, desesperanza y baja autoestima. Además, se ha investigado el rol de los factores gatillantes, tales como los eventos vitales estresantes, y la disponibilidad de métodos suicidas ${ }^{15,16,19-23}$. En nuestro país, en adolescentes se ha estudiado la relación de la suicidalidad con género, edad, depresión, desesperanza, autoestima, impulsividad, funcionamiento familiar, apoyo social y antecedentes de suicidalidad ${ }^{10-13}$.

En la última década y preferentemente a instancias de la OMS, se está dando importancia a la investigación epidemiológica de la conducta suicida, recomendándose realizar estudios de prevalencia, de tendencia y de factores de riesgo a nivel local, ya que la variabilidad observada entre los países parece ser reflejo de la verdadera naturaleza de dicha conducta más que de la variación en los métodos de investigación. Además, se recomienda testear modelos teóricos de factores de riesgos ${ }^{1,6}$.

En adolescentes, en relación con la conducta suicida, los trastornos psiquiátricos constituyen uno de los más fuertes predictores. Entre ellos destaca la depresión, el consumo de alcohol y drogas, y los problemas conductuales; siendo el trastorno depresivo el que cuenta con mayor evidencia ${ }^{15,16,24-26}$. Por otro lado, dentro de los rasgos de personalidad se cita la agresión, impulsividad, perfeccionismo y desesperanza como variables a considerar; de las cuales la desesperanza cuenta con más respaldo ${ }^{15,16}$. Escasos estudios han investigado la relación de la suicidalidad con otros factores relevantes en la adolescencia, tales como la soledad ${ }^{16,27}$. En adolescentes y jóvenes, se ha relacionado la soledad con ansiedad, síntomas depresivos, violencia y abuso de internet ${ }^{28-32}$.

Dada la evidencia, aunque aún no suficientemente investigada, de que el consumo de alcohol, la impulsividad y la soledad se relacionan con conducta suicida en adolescentes, y considerando la relevancia de estas variables en la adolescencia, el presente estudio pretende evaluar un modelo teórico conformado por dichas variables para la predicción de la suicidalidad (ideas suicidas más intento de suicidio). El objetivo es determinar la relación existente entre soledad, impulsividad, consumo de alcohol y suicidalidad; así como determinar la contribución relativa de dichas variables en la predicción de la suicidalidad en adolescentes estudiantes de enseñanza media de la comuna de Chillán.

\section{Sujetos y Métodos}

\section{Diseño de la investigación}

Estudio cuantitativo, transversal y correlacional.

\section{Participantes}

El universo lo constituyeron todos los estudiantes de $1^{\circ}$ a $4^{\circ}$ enseñanza media diurna, de colegios municipales, administrados por corporación, subvencionados y particulares, de la comuna de Chillán $(\mathrm{n}=13.825)$. El estudio se realizó en una muestra $(n=763)$, elegida por muestreo aleatorio simple, al azar, por etapas y estratificada por conglomerado, con nivel de confianza de $95 \%$ y margen de error máximo de 5\%. La edad estuvo comprendida entre 14 y 19 años con un promedio de 16 años (DE: 1,2). El 51,2\% eran de género femenino y $48,8 \%$ de género masculino.

\section{Instrumentos}

1. Escala de Suicidalidad: diseñada por Okasha et $\mathrm{al}^{33}$, autoadministrada, tipo Likert, formada por 4 ítems, graduado en cuatro categorías. Exploran ideas suicidas e intento de suicidio. La puntuación total oscila entre 0 y 12 puntos. La subescala de ideación suicida posee un punto de corte ( 5 puntos) para determinar riesgo de intento de suicidio. Validada en población adolescente chilena ${ }^{33}$, ha correlacionado significativamente con intentos suicidas, depresión, desesperanza, baja autoestima, impulsividad y bajo apoyo social ${ }^{10,12,13,33}$. En el presente estudio presentó un coeficiente alfa de Cronbach de 0,87 .

En el estudio actual la suicidalidad comprende las ideas suicidas más los intentos suicidas.

2. Escala de Soledad UCLA-III ${ }^{34}$ : compuesta por 20 ítems, autoaplicada, indaga sobre sentimientos de soledad asociados al tipo de relaciones 
Soledad, impulsividad, consumo de alcohol y suicidalidad en adolescentes - L. Salvo et al

que se establecen con otras personas. Con formato tipo Likert. La puntuación total oscila entre 20 y 80 puntos. En cuanto a su validez, en adolescentes chilenos, ha evidenciado correlaciones significativas negativas con apoyo social y positivas con depresión (Grant M, Sáenz C, Sifri C. Validación de la escala de soledad UCLA-3 en estudiantes de educación superior de la provincia de Concepción. Seminario de Tesis para optar al título de psicólogo, Universidad del Desarrollo, Concepción, Chile; 2009). En la presente investigación, alcanzó una confiabilidad en base al coeficiente alfa de Cronbach de 0,90.

3. Escala de Impulsividad de Barratt, versión 11 (BIS-11) ${ }^{35}$ : diseñada para evaluar impulsividad. Consta de 30 ítems agrupados en tres subescalas: impulsividad cognitiva, impulsividad motora e impulsividad no planeada. Cada ítem consta de cuatro opciones de respuesta. Es un instrumento autoaplicado. La puntuación total se obtiene de la suma de todos los ítems y puede oscilar entre 0 y 120 puntos. En nuestro medio ha sido validada en adolescentes, correlacionando positivamente con escala de impulsividad basada en conductas impulsivas descritas en DSM-IV, conductas impulsivas observadas por los profesores, conducta suicida y consumo de alcohol ${ }^{36}$. En el estudio actual obtuvo un coeficiente alfa de Cronbach fue de 0,77.

4. "Alcohol Use Disorders Identification Test" (AUDIT): cuestionario de auto-diagnóstico sobre riesgos en el uso de alcohol. Validado y empleado ampliamente en nuestro medio ${ }^{37}$. Consta de 10 ítems. Explora el consumo de alcohol del sujeto, así como los problemas derivados del mismo. Cada ítem cuenta con criterios operativos especificados para las puntuaciones correspondientes. En cada ítem, el valor de las respuestas oscila entre 0 y 4 (en los ítems 9 y 10 los valores posibles son tan sólo 0 , 2 y 4). Proporciona una puntuación total que se obtiene sumando las puntuaciones de los 10 ítems y que puede oscilar entre 0 y 40 . Existen puntos de corte diferenciados entre hombres y mujeres. En el presente estudio obtuvo un coeficiente alfa de Cronbach de 0,84.

\section{Procedimientos}

La información fue recolectada a través de un cuestionario que contenía los distintos instrumentos, anónimo, autoaplicado, y ensayado en muestra piloto. Para aplicarlo se solicitó autorización a directivos de educación y de los establecimientos educacionales. Para participar en el estudio se solicitó autorización a los padres/apoderados y alumnos, quienes aceptaron voluntariamente responder el cuestionario. La aplicación fue supervisada por profesionales de salud mental capacitados.

\section{Análisis de los datos}

Los datos se analizaron con el programa estadístico SPSS 15.0. Se determinó la confiabilidad de las escalas tipo Likert evaluando su consistencia interna con el coeficiente alfa de Cronbach. Se efectuó análisis descriptivo a través de distribución de frecuencias y estadísticos de resumen, análisis bivariado empleando prueba test de Student y coeficiente de correlación de Pearson, y análisis multivariado con análisis de regresión lineal múltiple. Se estableció un nivel de significación estadística de 0,05.

\section{Resultados}

Los resultados de este estudio revelan que en los últimos doce meses 19,1\% de los adolescentes estudiados había intentado suicidarse y $34,3 \%$ había presentado ideas suicidas de riesgo (5 o más puntos en subescala de ideación suicida de escala de suicidalidad). Los puntajes promedios en las

Tabla 1. Relación entre las variables en estudio y género, en adolescentes estudiantes de enseñanza media de la comuna de Chillán, Chile $(n=763)$

\begin{tabular}{|lcccc|}
\hline Variables & \multicolumn{2}{c}{ Género } & t & p \\
& Masculino M (DS) & Femenino M (DS) & \\
Suicidalidad & $2,81(2,95)$ & $4,37 \quad(3,50)$ & $-6,69$ & 0,000 \\
Soledad & $39,65(9,83)$ & $41,96(11,14)$ & $-3,04$ & 0,002 \\
Impulsividad & $49,85(13,95)$ & $48,75(15,76)$ & 1,03 & 0,306 \\
Consumo de alcohol & $4,15 \quad(5,18)$ & $2,54 \quad(3,98)$ & 4,77 & 0,000 \\
\hline
\end{tabular}


Soledad, impulsividad, consumo de alcohol y suicidalidad en adolescentes - L. Salvo et al

Tabla 2. Coeficientes y nivel de significación entre las variables predictoras y la suicidalidad, en adolescentes estudiantes de enseñanza media de la comuna de Chillán, Chile $(n=763)$

\begin{tabular}{|lcc|}
\hline Variables & r & p \\
\hline Edad & 0,12 & 0,001 \\
Soledad & 0,47 & 0,000 \\
Impulsividad & 0,30 & 0,000 \\
Consumo de alcohol & 0,18 & 0,000 \\
\hline
\end{tabular}

escalas empleadas fueron: Suicidalidad: 3,62 (DE: 3,33), Soledad: 40,84 (DE: 10,57), Impulsividad: 49,29 (DE: 14,90) y AUDIT: 3,32 (DE: 4,67).

Como se observa en la Tabla 1, se evidenció que las adolescentes de género femenino presentaron puntajes significativamente superiores en suicidalidad y soledad en comparación con los adolescentes masculinos. En cambio, estos últimos obtuvieron puntajes significativamente mayores en consumo de alcohol en comparación con las mujeres. Si bien los puntajes en impulsividad fueron más altos en varones que en mujeres, la diferencia no alcanzó significación estadística.

Por otro lado, se encontró que tanto la suicidalidad (r: 0,12; $\mathrm{p}<0,01$ ), como la soledad ( $\mathrm{r}$ : 0,14; $\mathrm{p}<0,001)$, impulsividad ( $\mathrm{r}: 0,10 ; \mathrm{p}<0,05)$ y consumo de alcohol $(\mathrm{r}: 0,25 ; \mathrm{p}<0,001)$ se relacionaron significativamente y en forma lineal directa con la edad de los adolescentes.

Para determinar si existía relación entre las variables independientes soledad, impulsividad y consumo de alcohol, y la variable dependiente suicidalidad, se llevó a cabo un análisis bivariado a través del coeficiente de correlación $r$ de Pearson. Los resultados, que se aprecian en la Tabla 2, permiten concluir que tanto la soledad como la impulsividad y el consumo de alcohol se relacionaron significativamente y en forma lineal directa con la suicidalidad.

Para someter a estudio el modelo planteado se efectuó un análisis de regresión lineal múltiple jerárquico entre la suicidalidad (variable criterio) y la soledad, impulsividad y consumo de alcohol (variables predictoras). Adicionalmente, se incorporaron las variables género y edad como variables de control para el modelo. En cuanto a la capacidad explicativa global del modelo, se obtuvo que el conjunto de variables explica $31,3 \%$ de la variable criterio $\left(\mathrm{R}^{2}\right.$ ajustado $\left.=0,313\right)$. Las variables sociodemográficas género y edad explican el 6,9\% de la suicidalidad $\left(\mathrm{R}^{2}\right.$ ajustado $\left.=0,069\right)$ y el grupo formado por las variables soledad, impulsividad y consumo de alcohol dan cuenta de $24,4 \%$ de la suicidalidad de los adolescentes estudiados $\left(\mathrm{R}^{2}\right.$ ajustado $=0,244$ ).

El análisis de varianza para determinar la significación estadística del conjunto de variables predictoras permitió concluir que existe relación lineal estadísticamente significativa entre el conjunto de variables predictoras y la variable criterio. Toda vez que obtiene un valor en el estadístico $\mathrm{F}=29,25(\mathrm{gl} 1=2, \mathrm{gl}=760), \mathrm{p}<0,001$ para el modelo $1 ; \mathrm{y} \mathrm{F}=70,32(\mathrm{gll}=5, \mathrm{gl} 2=757), \mathrm{p}<0,001$ para el modelo 2.

La Tabla 3 muestra la contribución de las variables predictoras a la capacidad explicativa del modelo y la importancia relativa de cada una sobre las variaciones en los valores de la suicidalidad. En estos análisis se aprecia que 4 de las 5 variables

Tabla 3. Coeficientes y niveles de significación para las variables incluidas en el modelo de regresión lineal $(n=763)$

\begin{tabular}{|c|c|c|c|c|c|}
\hline \multirow[t]{2}{*}{ Variables } & \multicolumn{2}{|c|}{ Coeficientes no estandarizados } & \multirow{2}{*}{$\begin{array}{c}\text { Coeficientes } \\
\text { estandarizados } \\
\beta\end{array}$} & \multirow[t]{2}{*}{$\mathbf{t}$} & \multirow[t]{2}{*}{ sr2 } \\
\hline & B & Error estándar & & & \\
\hline Género & 0,476 & 0,065 & 0,223 & $7,290 * * *$ & 0,047 \\
\hline Edad & 0,012 & 0,028 & 0,013 & 0,421 & 0,013 \\
\hline Soledad & 0,040 & 0,003 & 0,401 & $12,790 * * *$ & 0,147 \\
\hline Impulsividad & 0,012 & 0,002 & 0,164 & $5,000 * * *$ & 0,023 \\
\hline Consumo de alcohol & 0,128 & 0,028 & 0,154 & $4,625^{* * *}$ & 0,019 \\
\hline
\end{tabular}


predictoras incluidas en el modelo resultan ser estadísticamente significativas en su contribución a la explicación de los cambios en los valores de la suicidalidad, toda vez que género, soledad, impulsividad y consumo de alcohol presentan $\mathrm{p}<0,001$. Se observó además, que género femenino, soledad, impulsividad y consumo de alcohol tuvieron una relación directa con suicidalidad. La edad no se relacionó significativamente con suicidalidad.

Se observa también en la Tabla 3 que el orden de importancia en la contribución relativa a la explicación de la variable criterio fue primero soledad $(B=0,40 ;$ sr2 $=0,147)$, luego género $(B=0,22 ;$ sr2 $=0,047)$, seguido por impulsividad $(B=0,16 ; s r 2=0,023)$, y finalmente consumo de alcohol $(\mathrm{B}=0,15 ;$ sr2 $=0,019)$.

\section{Discusión}

Dado que las conductas suicidas en adolescentes tienen mayor prevalencia en la población general que en poblaciones clínicas, y considerando que hacia ese nivel deberían dirigirse preferentemente las estrategias preventivas ${ }^{16}$, el presente estudio tiene la fortaleza de aportar información proveniente de adolescentes de la comunidad. Además, no sólo indaga la relación de las variables en estudio con las ideas e intento de suicidio, sino que evalúa un modelo predictivo para la suicidalidad.

La principal debilidad metodológica de esta investigación es su diseño transeccional; si bien puede establecer correlaciones causales, no proporciona información sobre la estabilidad o la evolución de las relaciones a través del tiempo, como sí ocurriría con un diseño longitudinal ${ }^{38}$.

Aunque medir la prevalencia de la suicidalidad no era el objetivo de este estudio, llama la atención que la prevalencia en 12 meses de ideas suicidas $(34,3 \%)$ e intentos de suicidio (19,1\%) es superior a lo publicado en estudios internacionales; teniendo presente que las cifras de ideación suicida no son comparables dado el uso de diferentes definiciones. Al respecto, sería recomendable en investigaciones futuras en nuestro país utilizar ítems de ideación suicida e intento de suicidio provenientes de entrevistas estructuradas utilizadas en estudios de población en otros países para así facilitar la comparación internacional de nuestra realidad.

Concordante con otros estudios, la suicidalidad y la soledad fueron significativamente más fre- cuente en mujeres y el consumo de alcohol y la impulsividad mayor en varones (ésta última variable no alcanzó significación estadística). Así mismo, como se esperaba, de acuerdo con la literatura, la soledad, impulsividad y el consumo de alcohol se relacionó significativamente y en forma lineal directa con la suicidalidad en adolescentes ${ }^{16,23-26,30}$.

En relación con la soledad, futuros estudios longitudinales deberían indagar si dicha manifestación es correlato de otra condición psicopatológica, por ejemplo de síntomas depresivos, o si actúa independientemente y se mantiene en el tiempo.

El estudio elaboró un modelo para predecir el riesgo de suicidalidad a partir de las variables género y edad (introducidas como variables de control), soledad, impulsividad y consumo de alcohol. La capacidad explicativa global del modelo resultó aceptable $(31,8 \%)$, si se considera que la suicidalidad es multideterminada, y que el modelo no consideró predictores altamente correlacionados con ideas e intentos de suicidio en adolescentes como lo es la depresión, los intentos suicidas previos y la desesperanza. No se incorporaron esos factores por estar ampliamente respaldados por múltiples investigaciones.

A la luz de los hallazgos anteriores es importante evaluar en los adolescentes con trastornos depresivos y/o con trastornos por consumo de alcohol, la presencia de soledad e impulsividad, ya que estos rasgos contribuirían a aumentar considerablemente el riesgo suicida, más aún si existen otros factores de riesgo como conducta suicida previa, desesperanza y eventos estresantes.

Por tanto, los adolescentes con mayor soledad, más impulsivos y con mayor consumo de alcohol presentan más suicidalidad. Detectar y abordar las variables estudiadas podría ayudar a prevenir ideas e intentos suicidas.

\section{Referencias}

1. World Health Organization. Mental health suicide prevention (SUPRE). Disponible en: www.who.int/ mentl_health/prevention/suicide/suicideprevent/en/ index.html [Consultado el 24 de septiembre de 2011].

2. Organización Mundial de la Salud. Informe sobre la salud en el mundo 2001. Salud mental nuevos conocimientos, nuevas esperanzas. Ginebra: OMS; 2001. Disponible en: http://onsm.ces.edu.co/uploads/files/373302_whr01_es.pdf [Consultado el 25 de febrero de 2012]. 
3. Cash SJ, Bridge JA. Epidemiology of youth suicide and suicidal behavior. Curr Opin Pediatr 2009; 21: 613-9.

4. Pitman A, Krysinska K, Osborn D, King M. Suicide in young men. Lancet 2012; 379: 2383-92.

5. Patton GC, Coffey C, Sawyer SM, Viner RM, Haller DM, Bose K, et al. Global patterns of mortality in young people: a systematic analysis of population health data. Lancet 2009; 374: 881-92.

6. Nock M, Borges G, Bromet E, Cha C, Kessler R, Lee S. Suicide and suicidal behavior. Epidemiol Rev 2008; 30 (1): 133-54.

7. Waldrop AE, Hanson RF, Resnick HS, Kilpatrick DG, Naugle AE, Saunders BE. Risk factors for suicidal behavior among a national sample of adolescents: Implications for prevention. J Trauma Stress 2007; 20 (5): 869-79.

8. Zemaitiene N, Zaborskis A. Suicidal tendencies and attitude towards freedom to choose suicide among Lithuanian schoolchildren: results from three cross-sectional studies in 1994, 1998, and 2002. BMC Public Health 2005; 5: 83. Disponible en: http://www.biomedcentral. com/1471-2458/5/83 [Consultado el 25 de febrero de 2012].

9. Miauton L, Narring F, Michaud PA. Chronic illness, life style and emotional health in adolescence: results of a cross-sectional survey on the health of 15-20-year-olds in Switzerland. Eur J Pediatr 2003; 162 (10): 682-9.

10. Quinteros P, Grob F. Depresión y suicidalidad en una población no clínica de adolescentes. Bol Soc Psiquiat Neurol Infanc Adolesc 2003; 14 (1): 4-8.

11. Salvo L, Rioseco P, Salvo S. Ideación suicida e intento de suicidio en adolescentes de enseñanza media. Rev Chil Neuro-Psiquiatr 1998; 36: 28-34.

12. Salvo L, Melipillán R. Predictores de suicidalidad en adolescentes. Rev Chil Neuro-Psiquiatr 2008; 46: 115-23.

13. Ventura-Juncá R, Carvajal $C$, Undurraga $S$, Vicuña $P$, Egaña J, Garib M. Prevalencia de ideación e intento suicida en adolescentes de la Región Metropolitana de Santiago de Chile. Rev Med Chile 2010; 138: 309-15.

14. Florenzano R, Cáceres E, Valdés M, Calderón S, Santander S, Casassus M. Conductas de riesgo, síntomas depresivos, auto y heteroagresión en una muestra de adolescentes escolarizados en la Región Metropolitana de Santiago de Chile, 2007. Rev Chil Neuro-Psiquiat 2009; 47: 24-33.

15. American Psychiatric Association. Practice guideline for the assessment and treatment of patients with suicidal behaviors. Am J Psychiatry 2003; 160 (Suppl 11): 1-60.

16. Hawton K, Saunders KE, O'Connor RC. Self-harm and suicide in adolescents. Lancet 2012; 379: 2373-82.

17. Sourander A, Klomek AB, Niemela S, Haavisto A, Gy- llenberg D, Helenius $\mathrm{H}$, et al. Childhood predictors of completed and severe suicide attempts: findings from the Finnish 1981 Birth Cohort Study. Arch Gen Psychiatry 2009; 66 (4): 398-406.

18. Fortune S, Stewart A, Yadav V, Hawton K. Suicide in adolescents: using life charts to understand the suicidal process. J Affect Disord 2007; 100: 199-210.

19. Madge N, Hewitt A, Hawton K, de Wilde EJ, Corcoran P, Fekete S, et al. Deliberate self-harm within an international community sample of young people: comparative findings from the Child and Adolescent Self-harm in Europe (CASE) Study. J Child Psychol Psychiatry 2008; 49 (6): 667-77.

20. Nock MK, Borges G, Bromet EJ, Alonso J, Angermeyer $\mathrm{M}$, Beautrais A, et al. Cross-national prevalence and risk factors for suicidal ideation, plans and attempts. Br J Psychiatry 2008; 192 (2): 98-105.

21. Hargus E, Hawton K, Rodham K. Distinguishing between subgroups of adolescents who self-harm. Suicide Life Threat Behav 2009; 39 (5): 518-37.

22. O'Connor RC, Rasmussen S, Hawton K. Predicting deliberate self-harm in adolescents: a six month prospective study. Suicide Life Threat Behav 2009; 39 (4): 364-75.

23. O'Connor RC, Rasmussen S, Hawton K. Distinguishing adolescents who think about self-harm from those who engage in self-harm. Br J Psychiatry 2012; 200: 330-5.

24. Aseltine RH, Schilling EA, James A, Glanovsky JL, Jacobs D. Age variability in the association between heavy episodic drinking and adolescent suicide attempts: findings from a large-scale, school-based screening program. J Am Acad Child Adolesc Psychiatry 2009; 48 (3): 262-70.

25. Vijayakumar L, Kumar MS, Vijayakumar V. Substance use and suicide. Curr Opin Psychiatry 2011; 24 (3): 197 202.

26. King M, Semlyen J, Tai S, Killaspy H, Osborn D, Popelyuk $\mathrm{D}$, et al. A systematic review of mental disorder, suicide, and deliberate self-harm in lesbian, gay and bisexual people. BMC Psychiatry 2008; 8: 70. Disponible en: http://www.biomedcentral.com/1471-244X/8/70 [Consultado el 25 de febrero de 2012].

27. Johnson JG, Cohen P, Gould MS, Kasen S, Brown J, Brook JS. Childhood adversities, interpersonal difficulties, and risk for suicide attempts during late adolescence and early adulthood. Arch Gen Psychiatry 2002; 59: 741-9.

28. Fontaine RG, Yang C, Burks VS, Dodge KA, Price JM, Pettit GS, et al. Loneliness as a partial mediator of the relation between low social preference in childhood and anxious/depressed symptoms in adolescence. Dev Psychopathol 2009; 21 (2): 479-91.

29. Qualter P, Brown SL, Munn P, Rotenberg KJ. Childhood 
Soledad, impulsividad, consumo de alcohol y suicidalidad en adolescentes - L. Salvo et al

loneliness as a predictor of adolescent depressive symptoms: An 8-year longitudinal study. Eur Child Adolesc Psychiatry 2010; 19 (6): 493-501.

30. Lasgaard M, Goossens L, Elklit A. Loneliness, depressive symptomatology, and suicide ideation in adolescence: Cross-sectional and longitudinal analyses. J Abnorm Child Psychol 2011; 39: 137-50.

31. Kunst MJ, van Bon-Martens MJ. Examining the link between domestic violence victimization and loneliness in a Dutch community sample: A comparison between victims and nonvictims by type personality. J Fam Violence 2011; 26 (5): 403-10.

32. Kim J, LaRose R, Peng W. Loneliness as the cause and the effect of problematic internet use: The relationship between internet use and psychological well-being. Cyberpsychol Behav 2009; 12 (4): 451-5.

33. Salvo L, Melipillán R, Castro A. Confiabilidad, validez y punto de corte para escala de screening de suicidalidad en adolescentes. Rev Chil Neuro-Psiquiat 2009; 47: 1123.

34. Russel D, Peplau LA, Cutrona CE. The revised UCLA Loneliness Scale: Concurrent and discriminant validity evidence. J Pers Soc Psychol 1980; 39: 472-80.

35. Oquendo M, Baca-García E, Graver R, Morales M, Montalbán V, Mann J. Spanish adaptation of the Barratt Impulsiveness Scale (BIS). Eur J Psychiatry 2001; 15: 147-55.

36. Salvo L, Castro A. Validación de la escala de impulsividad de Barrat (BIS 11). Rev Chil Neuro-Psiquiatr 2011; 49 (Supl1): 97.

37. Alvarado M, Garmendia M, Acuña G, Santis R, Arteaga O. Validez y confiabilidad de la versión chilena del Alcohol Use Disorders Identification Test (AUDIT). Rev Med Chile 2009; 137: 1463-8.

38. Hernández R, Fernández C, Baptista M. Metodología de la investigación. México DF: Mc Graw Hill; 2010. 